\title{
OBSERVATIONS SUR LA BIOLOGIE DES PREMIERS STADES LARVAIRES D'ANGUILLICOLA CRASSUS, NÉMATODE PARASITE D'ANGUILLE
}

\author{
A. J. PETter, J. CASSONE, N. LE BELle
}

\begin{abstract}
RÉSUMÉ
Certains points de la biologie des premiers stades larvaires d'Anguillicola crassus, Nématode parasite de la vessie gazeuse des Anguilles, sont précisés : la durée de vie des jeunes larves libres du $2^{\mathrm{e}}$ stade dans l'eau est d'une quinzaine de jours à des températures variant de 18 à $29^{\circ} \mathrm{C}$, et d'environ quarante jours à $12^{\circ} \mathrm{C}$. Le développement jusqu'au $3^{\text {e }}$ stade larvaire a été obtenu expéri-

mentalement chez des Cyclopidae et chez l'Ostracode Cypria ophthalmica. A une température variant de 18 à $29^{\circ} \mathrm{C}$, les larves du $3^{\mathrm{e}}$ stade apparaissent à $\mathrm{J} 6$; à $12^{\circ} \mathrm{C}$, le développement est très lent et l'ébauche de la $2^{\mathrm{e}}$ mue s'observe seulement à $\mathrm{J} 62$. Des tentatives d'infestation du Branchiopode Cerodaphnia laticauda ont été négatives.
\end{abstract}

Summary: Observations on the biology of the early larval stages of Anguillicola crassus, a nematode parasite of anguillid eels.

Some data are given on the biology of the early larval stages of Anguillicola crassus, a nematode parasite found in the swimbladder of anguillid eels. The second-stage free larvae remained viable in water for about 15 days at a temperature of $18-29^{\circ} \mathrm{C}$, and for about 40 days at a temperature of $12^{\circ} \mathrm{C}$. Development up to the third larval stage was experimentally done in cyclopid copepods and in Cypria ophthalmica (Ostracoda). At a temperature varying from 18 to $29^{\circ} \mathrm{C}$, third-stage larvae first appeared on day 6. At $12^{\circ} \mathrm{C}$, development was very slow and start of the second molt was observed on day 62 . Infection of the branchiopod Cerodaphnia laticauda was attempted but was unsuccessful.
Le genre Anguillicola, Nématode parasite de la vessie gazeuse des Anguilles, est apparu il y a quelques années en Europe (Paggi et coll., 1982, Neumann, 1985), où il a pris une rapide extension. En particulier, l'espèce Anguillicola crassus Kuwahara, Niimi et Itagaki, 1974 a été identifiée dans divers pays (cf. Moravec et Taraschewski, 1988) et notamment en France (Dupont et Petter, 1988, Petter, Fontaine et Le Belle, 1989). Le cycle biologique de l'espèce a été étudié au Japon par Hirose, Sekino et Egusa, 1976, et en Europe par De Charleroy, Thomas et Belpaire, 1987, De Charleroy et coll., 1989, et Haenen et coll., 1989.

Dans un premier travail, nous avions décrit le développement des premiers stades larvaires chez un Cyclopidae de la région parisienne, Acanthocyclops robustus (Sars, 1863) (voir Petter, Fontaine et Le Belle, 1989). Nous cherchons ici à préciser certains points de la biologie des premiers stades larvaires : durée de vie des jeunes larves du $2^{\mathrm{e}}$ stade dans l'eau, possibilités de développement chez

Laboratoire des Vers associé au CNRS, Muséum national d'Histoire naturelle, 61, rue Buffon, F 75231 Paris Cedex 05.

Laboratoire de Physiologie générale et comparée, Laboratoire d'Endocrinologie comparée associé au CNRS, Muséum national d'Histoire naturelle, 7, rue Cuvier, F 75231 Paris Cedex 05.

Accepté le : 23 octobre 1989. d'autres Crustacés fréquents dans les eaux douces d'Europe, durée de développement des larves chez l'hôte intermédiaire à différentes températures.

\section{MATÉRIEL ET MÉTHODES}

Les œufs d'Anguillicola crassus proviennent des utérus de femelles mûres recueillies respectivement le 16 mai 1989 et le 7 juin 1989 dans la vessie gazeuse d'Anguilles argentées (Anguilla anguilla L.) originaires d'étangs du Nord de la France et conservées dans des aquariums d'eau courante au laboratoire. Les œufs sont placés dans des petits cristallisoirs emplis d'eau de mare, les uns dans une étuve à la température constante de $12^{\circ} \mathrm{C}$, les autres à la température du laboratoire, variant pendant la durée de l'expérience de $18^{\circ} \mathrm{C}$ la nuit à $29^{\circ} \mathrm{C}$ dans l'après-midi.

Des tentatives d'infestation ont été faites avec 3 groupes de Crustacés : $1-$ des Cyclopidae (Copépodes) non identifiés; $2-$ des Cypria ophthalmica (Jurine, 1820) (Ostracodes); 3 - des Cerodaphnia laticauda P.-E. Müller, 1867 (Branchiopodes).

Les Crustacés proviennent d'une mare d'un jardin de Lagnysur-Marne et de deux étangs situés à Isles-les-Villenoy et à Lesches (Seine-et-Marne). Ils sont placés dans des cristallisoirs contenant des œufs ou des larves écloses d'Anguillicola crassus : un cristallisoir avec des Cyclopidae est placé à l'étuve à $12^{\circ} \mathrm{C}$; les autres cristallisoirs sont placés à la température du laboratoire, les uns contenant une seule espèce de Crustacés, les autres plusieurs espèces mélangées; certaines expériences sont réalisées avec des larves ayant séjourné de 10 à 29 jours à $12^{\circ} \mathrm{C}$. 


\section{RÉSULTATS}

I - Durée de vie des larves dans L'Eau

a - A la température du laboratoire $\left(\begin{array}{lll}\left.18 \text { à } 29^{\circ} \mathrm{C}\right) \\ \text { - }\end{array}\right.$

La plupart des œufs sont éclos après 2 jours et les larves restent vivantes et s'agitent dans le fond du cristallisoir pendant une quinzaine de jours.

b $-A 12^{\circ} \mathrm{C}$

Les premières éclosions s'observent après 8 jours et la plupart des larves sont écloses seulement après 15 jours. Par contre, leur durée de vie est beaucoup plus longue, une quarantaine de jours pour la majorité des larves. Mises en contact avec des Crustacés après 29 jours à $12^{\circ} \mathrm{C}$, certaines d'entre elles au moins ont gardé la capacité de se développer chez l'hôte intermédiaire.

\section{II - Développement des laRves DANS les CyClopidae}

$\mathrm{a}-A 12^{\circ} \mathrm{C}$

Les Cyclopidae sont mis en contact avec les larves pendant 15 jours, puis transférés dans un cristallisoir dépourvu de larves. Ils sont examinés du $13^{\mathrm{e}}$ jour au $62^{\mathrm{e}}$ jour d'infestation; l'expérience est alors arrêtée car il ne reste plus de Cyclopidae vivants dans le cristallisoir.

Sur un total de 17 individus examinés, 16 ont été trouvés parasités; le nombre de larves présentes chez un hôte varie de 2 à une vingtaine; toutes les larves récoltées sont des $2^{\text {es }}$ stades; la croissance des larves est très faible pendant les 45 premiers jours, puis elle s'accélère entre le $45^{\mathrm{e}}$ et le $52^{\mathrm{e}}$ jour, et une des larves récoltées après 62 jours montre l'ébauche de la mue du $3^{\mathrm{e}}$ stade à son extrémité postérieure. Cependant, certaines larves ne montrent aucune croissance pendant toute la durée de l'expérience (fig. 1).
Trois Cyclopidae ont été retirés de l'étuve à $12^{\circ} \mathrm{C}$ après 45 jours et placés à la température du laboratoire; les larves grandissent alors rapidement et après 7 jours, la plupart sont au $3^{\mathrm{e}}$ stade.

$\mathrm{b}-A 18-29^{\circ} \mathrm{C}$

Six lots de Cyclopidae ont été mis en contact avec les larves. Sur un total de 29 spécimens examinés, 22 ont été trouvés parasités; le \% d'infestation varie de 57 à $80 \%$ suivant les lots. Le nombre de larves présentes chez un hôte varie de une à 12 . La première larve du $3^{\mathrm{e}}$ stade a été observée après 6 jours dans l'une des expériences, et après le $13^{\mathrm{e}}$ jour, la plupart des larves sont au $3^{\mathrm{e}}$ stade dans toutes les expériences.

Des larves du $3^{\mathrm{e}}$ stade sont parfois observées libres dans le fond des cristallisoirs; ces larves qui ont dû être libérées par la mort de leurs hôtes, restent vivantes pendant quelques jours dans l'eau.

\section{III - Infestation D’AUtres Crustacés}

Toutes les expériences ont été effectuées à la température du laboratoire.

\section{a - Cypria ophthalmica}

Huit lots de Cypria ophthalmica ont été mis en contact avec des larves, dont 3 dans des cristallisoirs ne contenant pas d'autres Crustacés, pour éliminer la possibilité d'infestation des Ostracodes par ingestion de larves ayant commencé leur développement chez des Cyclopidae.

Sur un total de 107 spécimens examinés, 28 ont été trouvés parasités, parmi lesquels 11 proviennent de cristallisoirs ne contenant pas de Cyclopidae; le \% d'infestation varie de 8 à $80 \%$ suivant les lots.

La croissance des larves est comparable à celle observée chez les Cyclopidae; un $3^{\mathrm{e}}$ stade larvaire est observé dès

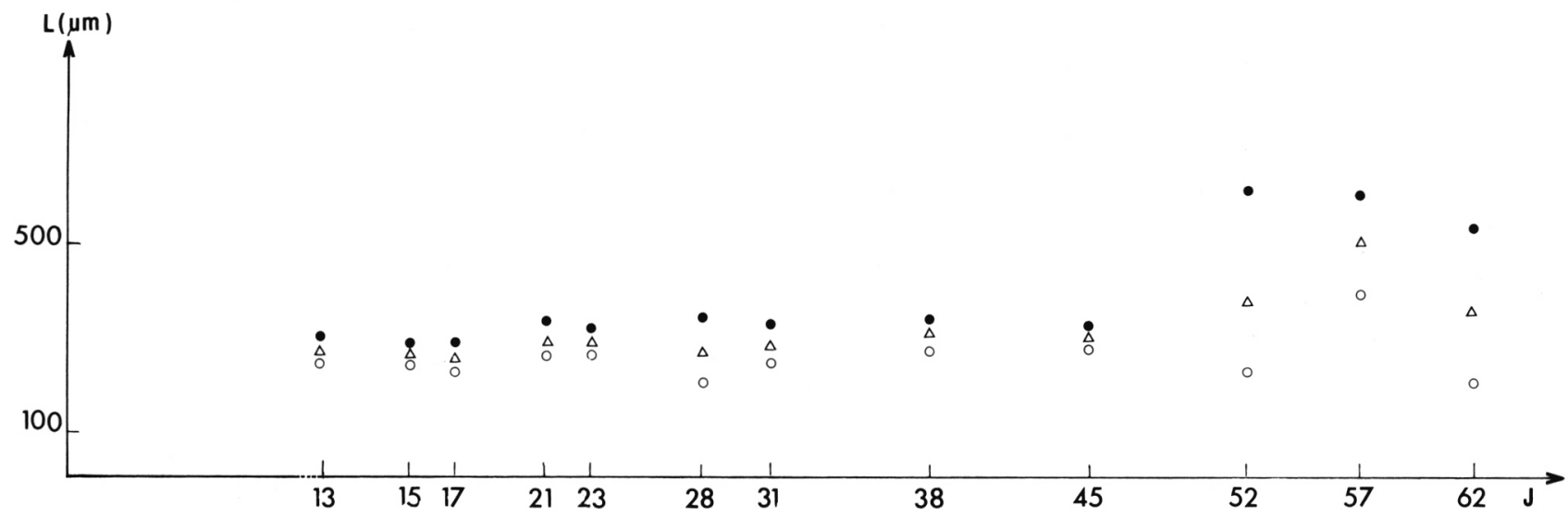

FIg. 1. - Croissance des larves d'Anguillicola crassus chez les Cyclopidae à $12^{\circ} \mathrm{C}$. J : nombre de jours d'infestation; L : longueur des larves; rond noir: longueur de la plus grande larve; rond blanc : longueur de la plus petite larve; triangle : longueur moyenne. 


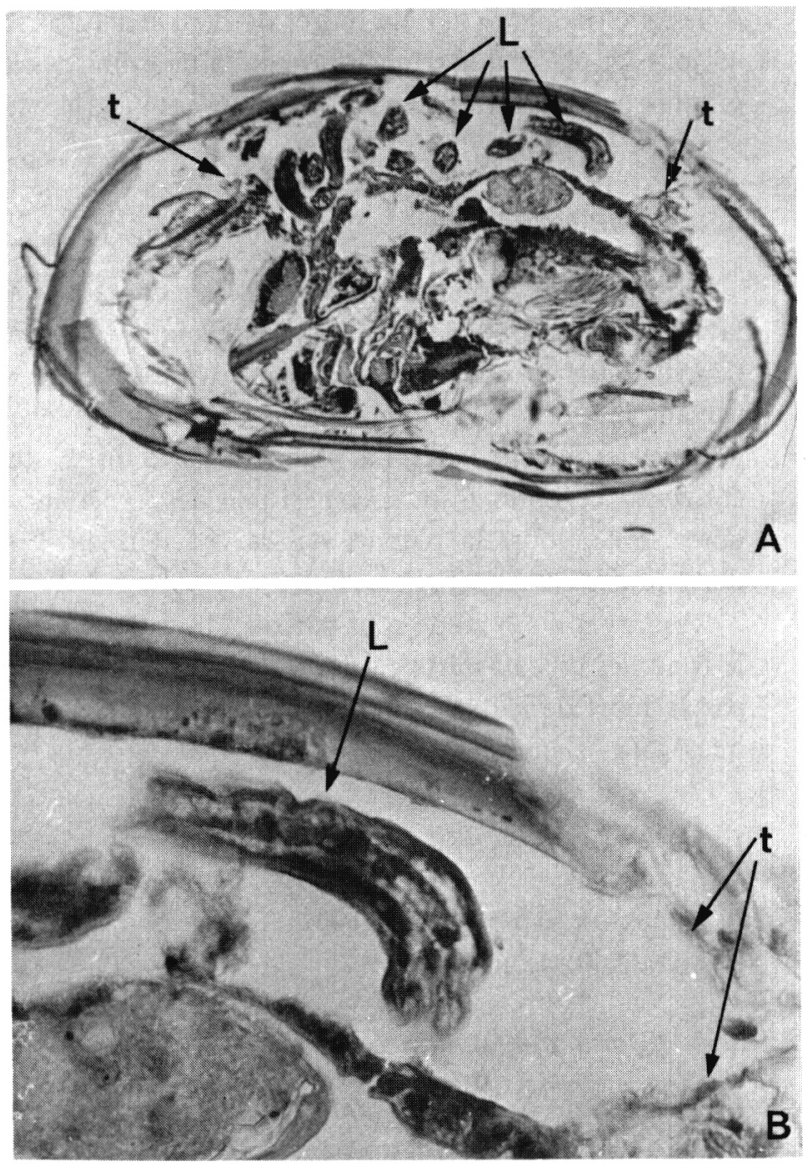

Fig. 2. - Coupes histologiques de Cypria ophthalmica parasitées par des larves d'Anguillicola crassus. A. corps entier, coupe sagittale; $\mathrm{B}$. détail de la région dorsale. $\mathrm{L}$ : larves; $t$ : tégument de l'Ostracode.

le $6^{\mathrm{e}}$ jour dans l'une des expériences, et à partir du $13^{\mathrm{e}}$ jour, la plupart des larves sont au $3^{\mathrm{e}}$ stade.

Le nombre de larves présentes chez un hôte varie de 1 à 6 ; les larves sont localisées dans l'haemocoele, principalement au voisinage de la charnière dorsale (fig. 2).

\section{b - Cerodaphnia laticauda}

Deux lots de Cerodaphnia laticauda ont été mis en contact avec des larves. Beaucoup sont morts dans les 3 premiers jours des expériences, de sorte que seulement 12 individus vivants ont pu être examinés : aucun n'a été trouvé parasité.

\section{CONCLUSIONS}

Nous pouvons tirer des expériences précédentes les principales conclusions suivantes :

1 - La durée de vie des jeunes larves du $2^{\mathrm{e}}$ stade dans l'eau dépend de la température : elles restent vivantes environ 40 jours à $12^{\circ} \mathrm{C}$ alors qu'à des températures plus élevées (18 à $29^{\circ} \mathrm{C}$ ), elles ne vivent qu'une quinzaine de jours. Ces chiffres sont légèrement supérieurs à ceux indiqués par De Charleroy et coll., 1987.

2 - Dans notre travail précédent, nous avions vu qu'à une température variant de 0 à $13^{\circ} \mathrm{C}$ les larves ne se développent pas et finissent par mourir dans les Copépodes, tandis qu'à une température de 20 à $22^{\circ} \mathrm{C}$, nous avions observé la $2^{\mathrm{e}}$ mue chez les Cyclopidae après 12 jours d'infestation; nous voyons ici qu'à une température plus élevée, atteignant $29^{\circ} \mathrm{C}$ dans l'après-midi, elle peut s'effectuer après seulement 6 jours d'infestation. Par contre, à une température constante de $12^{\circ} \mathrm{C}$, le développement est très lent : nous n'avons pas obtenu de $3^{\mathrm{e}}$ stade à $\mathrm{J} 62$.

3 - Le développement des larves peut s'effectuer chez les Ostracodes de l'espèce Cypria ophthalmica; cette espèce, commune dans les eaux douces françaises (Tétart, 1985 et communication personnelle) est donc susceptible de jouer un rôle dans la propagation du parasite.

Il est intéressant de constater que des Ostracodes paraissent constituer des hôtes intermédiaires non négligeables pour l'espèce Anguillicola crassus. En effet, les cycles évolutifs étudiés chez les Camallanina ont été effectués avec des Copépodes pour hôtes intermédiaires et les tentatives d'infestation avec d'autres Invertébrés ont généralement été négatives (Moravec, 1969, 1978, Stromberg et Crites, 1974, etc.); quelques cas seulement de développement jusqu'au stade infestant chez des Crustacés autres que les Copépodes sont connus : dans le genre Anguillicola, l'espèce A. globiceps Yamaguti, 1935 s'est développée chez des Cladocères (Haung, 1981, cité par Taraschewski et coll., 1987); Wellborn, 1970, a réalisé le développement de l'espèce $P h i$ lometra intraoculus chez un Ostracode (Cypridopsis sp.); Berry, 1986 a obtenu exceptionnellement le développement jusqu'au $3^{\mathrm{e}}$ stade de Spirocamallanus platycephali Hooper, 1983 chez un Amphipode, et il semble que les larves de Spirocamallanus cricotus Fusco et Overstreet, 1978 puissent se développer chez des Peneides (Overstreet, 1977, Fusco, 1980).

Remerciements. - Nous exprimons tous nos remerciements à Monsieur J. TÉTART qui a eu l'obligeance d'identifier les Ostracodes et les Cladocères et d'interpréter les coupes histologiques de Cypria ophthalmica.

\section{RÉFÉRENCES}

Berry G. N. : Life history of Spirocamallanus platycephali (Nematoda) in marine fish of Sillago spp. Parasitology-quo vadit? Handbook Sixth Intern. Congress Parasit., Brisbane, Australia, Program and Abstracts, 1986, 91.

De Charleroy D., Grisez L., Thomas K., Belpaire C., Ollevier F. : The life-cycle of Anguillicola crassa (Kuwahara, Niimi and Itagaki). Proc. F.H.S./A.F.S. Int. Fish Health Conference, Vancouver, 18-21 juillet 1988. J. Aquat. Animal Health (sous presse). 
De Charleroy D., Thomas K., Belpaire C. : Problems concerning the species determination, biology and diagnostical methods of Anguillicola, a swim-bladder nematode in the European eel (Anguilla anguilla L.) EIFAC Working Party on Eel, Bristol, U.K., 13-16 avril 1987, 7 p.

Dupont F., Petter A. J. : Anguillicola, une épizootie plurispécifique en Europe. Apparition de Anguillicola crassus (Nematoda, Anguillicolidae) chez l'Anguille européenne Anguilla anguilla en Camargue, sud de la France. Bull. Fr. Pêche Piscic., 1988, 308, 38-41.

Fusco A. C. : Larval development of Spirocamallanus cricotus (Nematoda: Camallanidae). Proc. Helminth. Soc. Wash., 1980, 47, 63-71.

Haenen O. L. M., Grisez L., De Charleroy D., Belpaire C., Ollevier F. : Migration of larvae of Anguillicola crassa after artificial infection in european eel Anguilla anguilla L. Proc. F.H.S./A.F.S. Int. Fish health Conference, Vancouver, 18-21 juillet 1988. J. Aquat. Animal Health (sous presse).

Hirose H., Sekino T., Egusa S. : Notes on the egg deposition, larval migration and intermediate host of the Nematode Anguillicola crassus parasitic in the swimbladder of eels. Fish Pathol., 1976, 11, 27-31 (en japonais, résumé anglais).

Moravec F.: Observations on the development of Camallanus lacustris (Zoega, 1776) (Nematoda: Camallanidae). Vestnick Cs. Spol. Zool. (Acta soc. zool. Bohemoslov.), 1969, 33, 15-33.

Moravec F. : The development of the nematode Philometra obturans (Prenant, 1886) in the intermediate host. Folia Parasitol., 1978, 25, 303-315.

Moravec F., Taraschewski, H. : Revision of the genus Anguillicola Yamaguti, 1935 (Nematoda: Anguillicolidae) of the swim- bladder of eels, including description of two new species, $A$. novaezelandiae sp. n. and A. papernai sp. n. Folia Parasitol., $1988,35,125-146$

Neumann W. : Schwimmblasenparasit Anguillicola bei Aalen. Fischer Teichwirt, 1985, 11, 322.

Overstreet R. M. : Parasites of some penaeid shrimps with emphasis on reared hosts. Aquaculture, 1973, 2, 105-140.

Paggi L., Orecchia P., Minervini R., Mattiucci S. : Sulla comparsa di Anguillicola australiensis Johnston et Mawson, 1940 (Dracunculoidea: Anguillicolidae) in Anguilla anguilla del lago di Bracciano. Parassitologia, 1982, 24, 139-144.

Petter A. J., Fontaine Y. A., Le Belle N. : Étude du développement larvaire de Anguillicola crassus (Dracunculoidea, Nematoda) chez un Cyclopidae de la région parisienne. Ann. Parasitol. Hum. Comp., 1989, 64, 347-355.

Stromberg P. C., Crites J. L. : The life-cycle and development of Camallanus oxycephalus Ward and Magath, 1916 (Nematoda: Camallanidae). J. Parasitol., 1974, 60, 117-124.

Taraschewski H., Moravec F., Lamah T., Anders K. : Distribution and morphology of two helminths recently introduced into European eel populations: Anguillicola crassus (Nematoda, Dracunculoidea) and Paratenuisentis ambiguus (Acanthocephala, Tenuisentidae). Dis. Aquat. Org., 1987, 3, 167-176.

Tétart J. : Description de la carapace des Ostracodes d'eau douce de France. II. Famille des Cyprididae (genres Cyclocypris, Cypria, Cypricercus, Eucypris). Spixiana, 1985, 8, 171-195.

Wellborn Jr. T. L. : The life history and pathology of the eye nematode, Philometra intraoculus sp. n., of bluegill (Lepomis macrochirus). Dissertation Abstracts International, 1971, $31 B$ (12), 7699-7700. 